\title{
Cardiac Imaging Methods for Chemotherapy-related Cardiotoxicity Screening and Related Radiation Exposure: Current Practice and Trends
}

\author{
BHASKAR C. KOLLA ${ }^{1}$, SAMIT SUNNY ROY ${ }^{2}$, SUE DUVAL ${ }^{2}$, \\ DANIEL WEISDORF ${ }^{1}$, UMA VALETI ${ }^{2}$ and ANNE BLAES ${ }^{1}$ \\ ${ }^{1}$ Division of Hematology, Oncology and Transplantation, \\ University of Minnesota Medical School, Minneapolis, MN, U.S.A.; \\ ${ }^{2}$ Cardiovascular Division, University of Minnesota Medical School, Minneapolis, MN, U.S.A.
}

\begin{abstract}
Background: Radiation exposure is a serious concern with usage of serial multigated acquisition (MUGA) scans (7.8mSv/study) for chemotherapy-related cardiotoxicity $(C R C)$ screening. The current practice with respect to the imaging modalities chosen for cardiotoxicity screening and related radiation exposure has not been studied. Materials and Methods: We performed a serial cross-sectional study from 2011 to 2014, evaluating the relative usage of the three imaging modalities for CRC screening. Results: MUGA scan usage decreased from $30.4 \%$ to $16.7 \%$, echocardiogram (Echo) utilization increased from $68.7 \%$ to $80.4 \%$ and cardiac magnetic resonance (CMR) usage increased from $0.9 \%$ to $2.9 \%$ in the 4-year period. Estimated total radiation exposure and secondary cancer risk can increase significantly in certain subgroups when MUGA scan is employed for serial cardiac imaging. Conclusion: Increased awareness of radiation risks from MUGA, as well as increasing focus on early detection of cardiotoxicity using Echo and CMR, are possible reasons behind the observed trends.
\end{abstract}

Serial cardiac imaging is performed to monitor decline in left ventricular ejection fraction (LVEF) in patients receiving cardiotoxic chemotherapy. Among adult survivors of pediatric malignancies who received anthracyclines, the incidence of systolic dysfunction was reported to range from $23 \%$ for patients with less than 10 years of follow-up to as high as $63 \%$ for those who had more than $500 \mathrm{mg} / \mathrm{m}^{2}$ of anthracycline at

This article is freely accessible online.

Correspondence to: Bhaskar C. Kolla, Division of Hematology, Oncology and Transplantation, University of Minnesota Medical School, 420 Delaware St SE, MMC 480, Minneapolis, MN, U.S.A. Tel: +1 6126251110,Fax: +1 6126256919, e-mail: bckolla@umn.edu

Key Words: Chemotherapy-related cardiotoxicity, modalities for cardiotoxicity screening, MUGA scan, radiation exposure, secondary cancer risk. more than 10 years of follow-up (1). In addition to anthracyclines, the risk of cardiomyopathy can rise into double digits with several other classes of agents, such as alkylating agents (up to $28 \%$ with cyclophosphamide), antimetabolites, monoclonal antibodies (2-28\% with trastuzumab) and small molecule tyrosine kinase inhibitors (up to $11 \%$ with sunitinib) $(2,3)$. Both American Heart Association (4) and American Society of Clinical Oncology (5) have general recommendations but do not specify the imaging methods that should be used to assess for cardiotoxicity, frequency of testing or duration of follow-up. Hence the practice is likely to be highly variable. Three commonly used imaging modalities for chemotherapy-related cardiotoxicity (CRC) screening include multigated acquisition (MUGA) scan, Echocardiography (Echo) and, more recently, cardiac magnetic resonance (CMR). Radiation exposure and secondary cancer risk are major concerns with serial MUGA scan usage (7.8 $\mathrm{mSv}$ per study) (6). In reviewing utilization trends of nuclear vs. echocardiographic stress testing in cardiology patients, the usage of nuclear testing increased from 1991 to 2004, plateaued, and, then, showed a rapid decline after 2006 (7). Publication of appropriate-use criteria in 2005 and increased awareness of radiation risks were cited as reasons for this observed trend $(7,8)$. The current practice of CRC screening with respect to the imaging modalities chosen has not been studied and is, thus, unknown.

We performed a serial cross-sectional study evaluating the relative usage of each of the three imaging modalities for CRC screening and any evolving trends based on patient and disease characteristics.

\section{Materials and Methods}

An electronic medical record review was performed for all adult patients at University of Minnesota Health and Fairview Hospitals and Clinics who received one or more of the four selected cardiotoxic drugs (doxorubicin, daunorubicin, idarubicin and trastuzumab) and underwent at least one cardiac imaging study 
Table I. Patients' demographic and clinical characteristics. Values are $n(\%)$.

\begin{tabular}{|c|c|c|c|c|}
\hline Characteristic & $\begin{array}{c}2011 \\
(n=313)\end{array}$ & $\begin{array}{c}2012 \\
(\mathrm{n}=514)\end{array}$ & $\begin{array}{c}2013 \\
(\mathrm{n}=559)\end{array}$ & $\begin{array}{c}2014 \\
(\mathrm{n}=518)\end{array}$ \\
\hline \multicolumn{5}{|l|}{ Age } \\
\hline$<30$ & $26(8.3)$ & $52(10.1)$ & $59(10.6)$ & $54(10.4)$ \\
\hline $30-39$ & $28(9.0)$ & $49(9.5)$ & $45(8.1)$ & $44(8.5)$ \\
\hline $40-49$ & $52(16.6)$ & $84(16.3)$ & $100(17.9)$ & 77 (14.9) \\
\hline $50-59$ & $94(30.0)$ & $131(25.5)$ & $141(25.2)$ & $132(25.9)$ \\
\hline $60-69$ & $68(21.7)$ & $123(23.9)$ & $132(23.6)$ & $143(27.6)$ \\
\hline$\geq 70$ & $45(14.4)$ & 75 (14.6) & $82(14.7)$ & $66(12.7)$ \\
\hline \multicolumn{5}{|l|}{ Gender } \\
\hline Male & $109(34.8)$ & $192(37.3)$ & $210(37.6)$ & $188(36.3)$ \\
\hline Female & $204(65.2)$ & $322(62.7)$ & $349(62.4)$ & $330(63.7)$ \\
\hline \multicolumn{5}{|l|}{ Ethnicity } \\
\hline White & $277(88.5)$ & $455(88.5)$ & $470(84.1)$ & $442(85.3)$ \\
\hline Black & $9(2.9)$ & $17(3.3)$ & $26(4.7)$ & $20(3.9)$ \\
\hline Asian & $7(2.2)$ & $14(2.7)$ & $14(2.5)$ & $16(3.1)$ \\
\hline Native American & $3(1.0)$ & $3(0.6)$ & 0 & $8(1.5)$ \\
\hline Pacific Islander & 0 & $2(0.4)$ & $2(0.4)$ & $1(0.2)$ \\
\hline Declined/Unknown & $17(5.4)$ & 0 & $41(7.3)$ & $31(6.0)$ \\
\hline \multicolumn{5}{|l|}{ Diagnosis } \\
\hline Breast cancer & $103(32.9)$ & $153(29.1)$ & $182(30.8)$ & $171(31.0)$ \\
\hline Lymphoma & $71(22.6)$ & $113(21.5)$ & $134(22.7)$ & $122(22.1)$ \\
\hline MDS/Leukemia/MPD & $46(14.6)$ & $74(14.1)$ & $76(12.9)$ & $79(14.3)$ \\
\hline Sarcoma & $32(10.2)$ & $53(10.1)$ & $61(10.3)$ & $59(10.7)$ \\
\hline Ovarian cancer & $11(3.5)$ & $36(6.8)$ & $33(5.6)$ & $21(3.8)$ \\
\hline Liver cancer & $17(5.4)$ & $29(5.6)$ & $38(6.4)$ & $32(5.8)$ \\
\hline Multiple myeloma & $14(4.8)$ & $18(3.4)$ & $11(1.9)$ & $14(2.5)$ \\
\hline Lung cancer & $4(1.3)$ & $8(1.5)$ & $4(0.7)$ & $3(0.5)$ \\
\hline Other/unknown or more than one diagnosis & $15(4.8)$ & $41(7.8)$ & $51(8.6)$ & $50(9.1)$ \\
\hline \multicolumn{5}{|l|}{ Drug received } \\
\hline Doxorubicin & $230(73.5)$ & $390(75.9)$ & $446(79.8)$ & $422(81.5)$ \\
\hline Trastuzumab & $42(13.4)$ & $55(10.7)$ & $49(8.8)$ & $39(7.5)$ \\
\hline Idarubicin & $32(10.2)$ & $47(9.1)$ & $42(7.5)$ & $39(7.5)$ \\
\hline Daunorubicin & $3(1.0)$ & $4(0.8)$ & $5(0.9)$ & $7(1.4)$ \\
\hline More than one drug & $6(1.9)$ & $18(3.5)$ & $17(3.0)$ & $11(2.1)$ \\
\hline
\end{tabular}

MDS, Myelodysplastic syndrome; MPD, myeloproliferative disease.

described. The data were collected partially for 2011 and for the whole of 2012-2014. Choice of cardiac imaging modality (MUGA, Echo or CMR) in patients receiving these four drugs was recorded. Current procedural terminology (CPT) and RxNorm Concept Unique Identifier (RXCUI) codes were used to identify the imaging study performed and the specific drug administered. The data were used to calculate the total number of cardiac imaging studies each year, stratified by imaging modality. Factors such as patient age, gender, disease subtype and type of cardiotoxic medication were assessed. The University of Minnesota Institutional Review Board reviewed and approved the study protocol.

Statistical analysis. Given the cross-sectional design, the same patient may appear in multiple years depending on their duration of need for CRC screening. Patients may have more than one diagnosis and, thus, the diagnosis data may not sum to the total number of patients in each year. Age was categorized into six groups, while all other data were categorical. The unit of measurement is an imaging
Table II. Total number of cardiac imaging studies and the corresponding percentage of echocardiogram (Echo), multigated acquisition (MUGA) and cardiac magnetic resonance (CMR) scans performed each year from 2011 to 2014

\begin{tabular}{lcccc}
\hline Imaging type & 2011 & 2012 & 2013 & 2014 \\
\hline MUGA & $178(30.4)$ & $236(23.7)$ & $214(20.0)$ & $169(16.7)$ \\
Echo & $402(68.7)$ & $745(74.7)$ & $841(78.5)$ & $811(80.4)$ \\
CMR & $5(0.9)$ & $16(1.6)$ & $17(1.6)$ & $29(2.9)$ \\
Total imaging studies & 585 & 997 & 1,072 & 1,009 \\
\hline
\end{tabular}

study for the trend analysis. The Cochran-Armitage test was used to test for significance of trend in imaging modality usage across study years. All statistical analyses were performed using SAS version 9.3 (SAS Institute Inc., Cary, NC, USA). 
Table III. Number of imaging studies per patient over 4 years, $n(\%)$, stratified by drug and disease categories.

\begin{tabular}{|c|c|c|c|c|c|}
\hline & 1 & 2 & 3 & 4 & 5 or more \\
\hline Trastuzumab & $10(11.6)$ & 10 (11.6) & $12(14)$ & $14(16.3)$ & $40(46.5)$ \\
\hline Doxorubicin & 419 (40) & $255(24.4)$ & 120 (11.5) & $89(8.5)$ & 163 (15.6) \\
\hline Idarubicin & $31(27.2)$ & $31(27.2)$ & $27(23.7)$ & $9(7.9)$ & $16(14)$ \\
\hline Daunorubicin & $3(27.3)$ & $3(27.3)$ & $0(0)$ & $1(9.1)$ & $4(36.3)$ \\
\hline More than one drug & $0(0)$ & $6(22.2)$ & $3(11.1)$ & $3(11.1)$ & $15(55.6)$ \\
\hline Breast cancer & $97(25.1)$ & $62(16.1)$ & $57(14.8)$ & $47(12.2)$ & $123(31.8)$ \\
\hline Lymphoma & $106(33.9)$ & $101(32.3)$ & $33(10.5)$ & $24(7.7)$ & $49(15.6)$ \\
\hline Leukemia & $47(24.6)$ & $55(28.8)$ & 35 (18.3) & $22(11.5)$ & $32(16.8)$ \\
\hline Sarcoma & $84(56)$ & $30(20)$ & $11(7.3)$ & $8(5.3)$ & $17(11.3)$ \\
\hline All other & $129(52.9)$ & $57(23.4)$ & $26(10.7)$ & $15(6)$ & $17(7)$ \\
\hline All patients & $463(36)$ & $305(23.8)$ & $162(12.6)$ & $116(9)$ & $238(18.5)$ \\
\hline
\end{tabular}

\section{Results}

There were a total of 1,284 patients who received one or more of the four drugs during the four years of study. Demographic and clinical characteristics are shown in Table I. Doxorubicin was the most commonly used drug followed by trastuzumab. Breast cancer and lymphoma were the top two diagnoses.

Over the study period, a total of 3,663 cardiac imaging studies were performed in this patient population (Table II). The usage of MUGA declined from $30.4 \%$ in 2011 to $16.7 \%$ in 2014. Concomitantly, the usage of Echo increased from $68.7 \%$ to $80.4 \%$ and CMR usage increased from $0.9 \%$ to $2.9 \%$. The trends for declining usage of MUGA $(p<0.001)$ and increasing usage of Echo ( $p=0.044)$ were statistically significant. A trend of increasing usage of CMR was also observed $(p=0.005)$, although the absolute number for CMR was small. Number of studies per patient ranged from 1 to 11 per year and 1 to 21 over 4 years $($ median=2) $(1,4)$ (not shown). Forty six percent of patients who received trastuzumab and $32 \%$ of breast cancer patients underwent 5 or more studies over the 4 years, compared to only $18 \%$ for all patients in the study group (Table III). These two subgroups also saw the largest decline in MUGA usage over the 4 years with $10 \%$ or less MUGA use in 2014 compared to $16 \%$ for the total group (Table IV).

\section{Discussion}

MUGA scan usage is declining and it is mostly replaced by Echocardiography. All three trends for declining MUGA usage and increasing Echo and CMR usage were seen consistently across all disease subtypes, types of chemotherapy, sex and age groups (Table IV). Although we cannot identify all the reasons for this decline in usage of MUGA scans, increased awareness of radiation risks is likely a notable contributor. The radiation exposure from a single MUGA scan is about
$7.8 \mathrm{mSv}$ (6). This is equivalent to 2.5 times the yearly background radiation in United States $(3 \mathrm{mSv})$. For example, in the case of adjuvant trastuzumab use in a woman with breast cancer, CRC screening is recommended prior to initiation of therapy, every 3 months during therapy and every 6 months for two years after completion of therapy per the manufacturer prescribing information (requiring total of 9 scans) (9). The Canadian Trastuzumab Working Group recommends screening during therapy for a minimum of 5 scans, while stating no evidence exists for continued screening following completion of therapy (10). However, ongoing screening may be necessary in patients who received anthracyclines due to their late cardiac effects (1). If we assume a woman with breast cancer who undergoes trastuzumab-based adjuvant chemotherapy and receives 9 MUGA scans for CRC screening and one positron emission tomography-computed tomography (PET-CT) for staging, the radiation exposure is approximately $100 \mathrm{mSv}(70 \mathrm{mSv}$ for MUGA scans and 25-30 mSv for PET-CT) (6). Using BEIR VII estimates, exposure to $100 \mathrm{mSv}$ of radiation in a 40 -yearold has additional lifetime risk of 1,300 cases of cancer per 100,000 women (11). Typically, this patient (depending on the stage of the disease) may also receive chest wall radiation and, possibly, more CRC screening for the anthracycline part of the adjuvant regimen plus additional surveillance imaging during the disease course, thereby greatly increasing the radiation exposure and secondary cancer risk. Using other imaging methods, such as echo-based techniques instead of MUGA, could reduce the total radiation burden significantly.

The number and frequency of cardiac imaging studies and duration of follow-up required for $\mathrm{CRC}$ monitoring depends on the cardiotoxicity risk. The incidence of cardiotoxicity can vary depending on patients' characteristics, such as age, duration of survival after the inciting agent, presence of other co-morbidities like hypertension, cardiovascular disease, pre- 
Table IV. Imaging study trends stratified by selected variables.

\begin{tabular}{|c|c|c|c|c|}
\hline Variable - n (\%) & 2011 & 2012 & 2013 & 2014 \\
\hline \multicolumn{5}{|l|}{ Breast cancer } \\
\hline MUGA & $55(29.8)$ & $63(18.4)$ & $72(16.8)$ & $40(10.8)$ \\
\hline Echo & $127(69.0)$ & $272(79.7)$ & 345 (80.7) & $316(86.1)$ \\
\hline CMR & $2(0.9)$ & $6(1.7)$ & $10(2.3)$ & $11(2.99)$ \\
\hline Total & 184 & 341 & 427 & 367 \\
\hline \multicolumn{5}{|l|}{ Lymphoma } \\
\hline MUGA & $29(22.1)$ & $41(20.6)$ & $38(14.8)$ & 37 (15.4) \\
\hline Echo & $102(77.8)$ & $153(76.8)$ & $213(83.2)$ & $194(81.1)$ \\
\hline CMR & $0(0)$ & $5(2.4)$ & $5(2)$ & $8(3.3)$ \\
\hline Total & 131 & 199 & 256 & 239 \\
\hline \multicolumn{5}{|l|}{ Doxorubicin } \\
\hline MUGA & $123(30.2)$ & $174(24.2)$ & 154 (18.9) & 137 (17.4) \\
\hline Echo & $281(69.2)$ & $533(74.1)$ & $642(78.9)$ & $623(79.3)$ \\
\hline CMR & $2(0.4)$ & $12(1.6)$ & $17(2.0)$ & $25(3.1)$ \\
\hline Total & 406 & 719 & 813 & 785 \\
\hline \multicolumn{5}{|l|}{ Trastuzumab } \\
\hline MUGA & $32(34.7)$ & $19(14.5)$ & $20(15.5)$ & $8(8.6)$ \\
\hline Echo & $58(63)$ & $108(82.4)$ & $109(84.5)$ & $83(90.2)$ \\
\hline CMR & $2(2.1)$ & $4(3)$ & 0 & $1(1.2)$ \\
\hline Total & 92 & 131 & 129 & 92 \\
\hline \multicolumn{5}{|l|}{ Female } \\
\hline MUGA & $134(34.7)$ & $143(22.6)$ & $144(20.4)$ & $104(16)$ \\
\hline Echo & 247 (63.9) & $480(76.2)$ & $549(77.8)$ & $527(81.2)$ \\
\hline CMR & $5(1.2)$ & $7(1.1)$ & $12(1.7)$ & $18(2.7)$ \\
\hline Total & 386 & 630 & 705 & 649 \\
\hline \multicolumn{5}{|l|}{ Male } \\
\hline MUGA & $44(22.1)$ & $93(25.3)$ & $70(19)$ & $65(18)$ \\
\hline Echo & $155(77.8)$ & $265(72.2)$ & $292(79.5)$ & $284(78.8)$ \\
\hline CMR & 0 & $9(2.4)$ & $5(1.4)$ & $11(3)$ \\
\hline Total & 199 & 367 & 367 & 360 \\
\hline \multicolumn{5}{|l|}{ Age $51-60$} \\
\hline MUGA & $52(30.5)$ & $55(22.9)$ & $65(22.5)$ & $51(16.1)$ \\
\hline Echo & $115(67.6)$ & $180(75)$ & $223(77.4)$ & $254(80.3)$ \\
\hline CMR & $3(1.7)$ & $5(2)$ & 0 & $11(3.4)$ \\
\hline Total & 170 & 240 & 288 & 316 \\
\hline \multicolumn{5}{|l|}{ Age $61-70$} \\
\hline MUGA & $45(36.3)$ & $64(26.3)$ & $51(23.9)$ & $31(17.2)$ \\
\hline Echo & $77(62.1)$ & 174 (71.6) & $158(74.2)$ & $143(79.4)$ \\
\hline CMR & $2(1.6)$ & 52) & $4(1.9)$ & $6(3.4)$ \\
\hline Total & 124 & 243 & 213 & 180 \\
\hline
\end{tabular}

MUGA, Multigated acquisition; Echo, echocardiogram; CMR, cardiac magnetic resonance.

existing left ventricular dysfunction and on treatment characteristics, such as dose of the drug, associated mediastinal radiation or usage of other cardiotoxic drugs. The majority of patients in our study underwent only 1 or 2 cardiac imaging studies during the 4 years (Table III). However, in patients who require frequent monitoring and a long monitoring period, such as in breast cancer patients who receive trastuzumab, the number of imaging studies was higher. Forty-six percent of patients who received trastuzumab and $32 \%$ of breast cancer patients had 5 or more studies over 4 years compared to only $18 \%$ of all patients in the study group (Table III). Also $55 \%$ of patients who received more than one cardiotoxic drug underwent 5 or more studies during the 4 years. As can be seen in Table IV, the breast cancer and trastuzumab subgroups saw the largest decline in MUGA scan usage over the four years and have the lowest relative usage of MUGA in 2014, likely reflecting the clinically appropriate drive to lower their total radiation burden.

Other factors contributing to these observed trends in imaging modality choice could be the more comprehensive anatomic and functional detail available using Echo and CMR, as well as their capability for early detection of cardiotoxicity. A decline in left ventricular ejection fraction may be a late manifestation of cardiotoxicity (12) and there has been an increasing focus on early detection of CRC using techniques, such as strain monitoring using Echo (13) and T2-weighted imaging for myocardial edema using CMR (14). There have been algorithms proposed to incorporate these techniques into the CRC screening protocols (15). It is essential to point out here that 2D Echo for serial LVEF monitoring has a high test-retest variability that is reported to be anywhere from $11 \%$ to as high as $17 \%$ (16). This raises the odds that a drop in LVEF of $10 \%$ detected using 2D Echo could be due to a sampling error (17). This can be improved by using a higher LVEF cut-off threshold by 2D Echo of $60 \%$ instead of $55 \%$ (18), by using contrast for better delineation of endocardial borders (interobserver variability of 9.6\%) (19) or by using 3D Echo (interobserver variability of $4.9 \%)(20)$.

This study suffers certain limitations. The study population is from a single health care delivery system in the Minneapolis-St. Paul Metropolitan area and vicinities in the United States. Therefore, this practice may not be representative of the general population. While there could be other indications for cardiac imaging in cancer patients, we assumed that the majority of cardiac imaging studies in this population were performed for CRC screening. Despite these limitations, we report the current practice trends over 4 years in a system where there was broad availability and consistent access to all the three imaging modalities.

\section{Conclusion and Clinical Implications}

MUGA usage has declined and Echo and CMR usages have increased in monitoring for CRC over the 4 years from 2011 to 2014. This snapshot of a current practice may reflect an increased awareness among patients and physicians of radiation risks associated with serial MUGA scans and, possibly, also the increasing focus on early detection of CRC using Echo and CMR. Estimated radiation exposure and secondary cancer risk could be significant in certain patient subgroups that require frequent monitoring and a prolonged treatment course when serial MUGA scan is employed for 
CRC screening. Hence the choice of imaging modality for cardiotoxicity screening may present an area of opportunity to lower total radiation burden among such patients.

\section{Conflicts of Interest}

None of the Authors have any conflicts of interest.

\section{References}

1 Steinherz LJ, Steinherz PG, Tan CT, Heller G and Murphy ML: Cardiac toxicity 4 to 20 years after completing anthracycline therapy. JAMA 266: 1672-1677, 1991.

2 Raschi E and De Ponti F: Cardiovascular toxicity of anticancertargeted therapy: Emerging issues in the era of cardio-oncology. Internal and emergency medicine 7: 113-131, 2012.

3 Chu TF, Rupnick MA, Kerkela R, Dallabrida SM, Zurakowski D, Nguyen L, Woulfe K, Pravda E, Cassiola F, Desai J, George S, Morgan JA, Harris DM, Ismail NS, Chen JH, Schoen FJ, Van den Abbeele AD, Demetri GD, Force $\mathrm{T}$ and Chen $\mathrm{MH}$ : Cardiotoxicity associated with tyrosine kinase inhibitor sunitinib. Lancet 370: 2011-2019, 2007.

4 Hunt SA: ACC/AHA 2005 guideline update for the diagnosis and management of chronic heart failure in the adult: A report of the American College of Cardiology/American Heart Association Task Force on Practice Guidelines (Writing Committee to Update the 2001 Guidelines for the Evaluation and Management of Heart Failure). J Am College Cardiol 46: e1-82, 2005.

5 Carver JR, Shapiro CL, Ng A, Jacobs L, Schwartz C, Virgo KS, Hagerty KL, Somerfield MR, Vaughn DJ and Panel ACSE: American Society of Clinical Oncology clinical evidence review on the ongoing care of adult cancer survivors: Cardiac and pulmonary late effects. J Clin Oncol 25: 3991-4008, 2007.

6 Mettler FA Jr., Huda W, Yoshizumi TT and Mahesh M: Effective doses in radiology and diagnostic nuclear medicine: A catalog. Radiology 248: 254-263, 2008.

7 Jouni H, Askew JW, Crusan DJ, Miller TD and Gibbons RJ: Temporal trends of single-photon emission computed tomography myocardial perfusion imaging in patients without prior coronary artery disease: A 22-year experience at a tertiary academic medical center. Am Heart J 176: 127-133, 2016.

8 McNulty EJ, Hung YY, Almers LM, Go AS and Yeh RW: Population trends from 2000-2011 in nuclear myocardial perfusion imaging use. JAMA 311: 1248-1249, 2014.

9 Genentech: Herceptin prescribing information- https:// www.gene.com/download/pdf/herceptin_prescribing.pdf. Downloaded 12-1-16.

10 Mackey JR, Clemons M, Cote MA, Delgado D, Dent S, Paterson A, Provencher L, Sawyer MB and Verma S: Cardiac management during adjuvant trastuzumab therapy: Recommendations of the Canadian Trastuzumab Working Group. Curr Oncol (Toronto, Ont) 15: 24-35, 2008.
11 National Research Council Committee on Health Effects of Exposure to Low Levels of Ionizing R: In: Health Effects of Exposure to Low Levels of Ionizing Radiations: Time for Reassessment? Washington (DC): National Academies Press (US), 1998.

12 Ewer MS and Lenihan DJ: Left ventricular ejection fraction and cardiotoxicity: Is our ear really to the ground? J Clin Ooncol 26: 1201-1203, 2008.

13 Sawaya H, Sebag IA, Plana JC, Januzzi JL, Ky B, Cohen V, Gosavi S, Carver JR, Wiegers SE, Martin RP, Picard MH, Gerszten RE, Halpern EF, Passeri J, Kuter I and ScherrerCrosbie M: Early detection and prediction of cardiotoxicity in chemotherapy-treated patients. Am J Cardiol 107: 1375-1380, 2011.

14 Meyersohn NM, Pursnani A and Neilan TG: Detection of cardiac toxicity due to cancer treatment: Role of cardiac MRI. Curr Options Cardiovasc Med 17: 396-405, 2015.

15 Kerkhove D, Fontaine C, Droogmans S, De Greve J, Tanaka K, Van De Veire $\mathrm{N}$ and Van Camp G: How to monitor cardiac toxicity of chemotherapy: Time is muscle! Heart 100: 1208$1217,2014$.

16 Van Royen N, Jaffe CC, Krumholz HM, Johnson KM, Lynch PJ, Natale D, Atkinson P, Deman P and Wackers FJ: Comparison and reproducibility of visual echocardiographic and quantitative radionuclide left ventricular ejection fractions. Am J Cardiol 77: 843-850, 1996.

17 Mor-Avi V and Lang RM: Is echocardiography reliable for monitoring the adverse cardiac effects of chemotherapy? J Am College Cardiol 61: 85-87, 2013.

18 Armstrong GT, Plana JC, Zhang N, Srivastava D, Green DM, Ness KK, Daniel Donovan F, Metzger ML, Arevalo A, Durand JB, Joshi V, Hudson MM, Robison LL and Flamm SD: Screening adult survivors of childhood cancer for cardiomyopathy: Comparison of echocardiography and cardiac magnetic resonance imaging. J Clin Oncol 30: 2876-2884, 2012.

19 Malm S, Frigstad S, Sagberg E, Larsson H and Skjaerpe T: Accurate and reproducible measurement of left ventricular volume and ejection fraction by contrast echocardiography: A comparison with magnetic resonance imaging. J Am College Cardiol 44: 1030-1035, 2004.

20 Thavendiranathan P, Grant AD, Negishi T, Plana JC, Popovic ZB and Marwick TH: Reproducibility of echocardiographic techniques for sequential assessment of left ventricular ejection fraction and volumes: Application to patients undergoing cancer chemotherapy. J Am College Cardiol 61: 77-84, 2013. 